\title{
Epitaxial Growth of ZnO Monolayer on Graphene: The Thinnest Metal Oxide Semiconductor
}

Hyo-Ki Hong ${ }^{1}$, Jongyeong Lee ${ }^{1}$, Na Yeon Kim ${ }^{1,2}$, Seoungwoo Son ${ }^{1}$, Jung Hwa Kim ${ }^{1}$, Rolf Erni ${ }^{3}$ and Zonghoon Lee Le, $^{1, *}$

1. School of Materials Science and Engineering, Ulsan National Institute of Science and Technology (UNIST), Ulsan 44919, S. Korea

2. Center for Multidimensional Carbon Materials, Institute for Basic Science (IBS), Ulsan 44919, S. Korea

3. Electron Microscopy Center, Empa - Swiss Federal Laboratories for Materials Science and Technology, CH-8600 Dübendorf, Switzerland

Heteroepitaxial growth of metal oxide semiconductors on two-dimensional layered nanomaterials, combining wide band gap and high carrier density, has become a new integration method for fabricating electronic devices [1]. Among semiconductor materials, zinc oxide has been used in novel transparent electronic devices as forms of epitaxial layer on graphene [2, 3]. Thermodynamically, hexagonal wurtzite $\mathrm{ZnO}$ is the common form [2]. The wurtzite structure of $\mathrm{ZnO}$ can be transformed to a planar $\mathrm{ZnO}$ monolayer in which $\mathrm{Zn}$ and $\mathrm{O}$ atoms reside in a trigonal planar coordination, instead of the bulk tetrahedral configuration formed when $\mathrm{ZnO}$ is thinned down to a few atomic layers [4]. Since $\mathrm{ZnO}$ monolayers on graphene can have many applications in electronic devices, growth of thin $\mathrm{ZnO}$ layers on graphene has been studied extensively [5]. Here, we provide experimental evidence for the epitaxial growth of a $\mathrm{ZnO}$ monolayer on graphene using atomic resolution transmission electron microscopy along with the corresponding image simulations and first principles calculations. Furthermore, we demonstrate through in situ observation the atom-by-atom growth of zinc and oxygen at the zigzag edge of the $\mathrm{ZnO}$ monolayer on graphene. In addition, we demonstrate the presence of 2-3 nm quantum dots of the epitaxial $\mathrm{ZnO}$ monolayer grown by atomic layer deposition (ALD). Unlike conventional bulk $\mathrm{ZnO}, \mathrm{ZnO}$ quantum dots have potential applications in nanoscale devices, such as photonic and electronic devices, due to the quantum confinement effect [6].

Figure 1 shows a $\mathrm{ZnO}$ monolayer grown on pristine graphene and the UV/ozone-treated graphene after 20 ALD cycles. Figure 1a shows the $\mathrm{ZnO}$ deposited on the pristine graphene after 20 ALD cycles. The red region indicates the crystallized $\mathrm{ZnO}$ monolayer. Figure $1 \mathrm{~b}$, however, shows larger size of $\mathrm{ZnO}$ crystals epitaxially grown on the UV/ozone-treated hydrophilic graphene after 20 ALD cycles. The ZnO coverage is much larger on the UV/Ozone-treated graphene. The misorientation angle of $0^{\circ}$ is the most common.

Figure 2 shows results of band gap measurements with electron energy loss spectroscopy in the scanning transmission electron microscopy for $\mathrm{ZnO}$ grown on graphene with different ALD cycles. The results display higher band gap energy for smaller $\mathrm{ZnO}$ nanoclusters. For example, a ZnO grown with 10 ALD cycles displays a band gap of $4.0 \mathrm{eV}$, whereas a $\mathrm{ZnO}$ grown with 200 ALD cycles exhibits a band gap of $3.25 \mathrm{eV}$, which is close to the bulk $\mathrm{ZnO}$ value. The observed gradual spectral shift in the band edge with the ALD cycles can be attributed to the expected quantum confinement effect [6].

We demonstrate the formation of $\mathrm{ZnO}$ monolayer on graphene, which is the thinnest heteroepitaxial layer of semiconducting oxide on graphene. The optimized UV/ozone treatment enhances the 
hydrophilic surface property of the graphene. Moreover, we experimentally determine that the monolayer $\mathrm{ZnO}$ on graphene has a wide band gap of up to $4.0 \mathrm{eV}$, which is different from that of other $\mathrm{ZnO}$ nanostructures, due to the quantum confinement effect and the crystallographic structure. The heteroepitaxial stack of the thinnest two-dimensional oxide semiconductors on graphene has potential for future electronic device applications. This study can lead to a new class of two-dimensional heterostructures including semiconducting oxides formed by highly controlled epitaxial growth through a deposition route [7].

\section{References:}

[1] K. Chung et al., Science 330 (2010), p. 655-657.

[2] S. J. Pearton et al., Superlattice and Microstructures 34 (2003), p. 3-32.

[3] Z. L. Wang. et al., Journal of Physics: Condensed Matter 16 (2004), p. R829-R858.

[4] Z. C. Tu. et al., Physical Review B 74 (2006), p. 035434.

[5] H. T. Quang. et al., ACS Nano 9 (2015), p. 11408-11413.

[6] H. K. Hong et al., Nano Letters 17 (2017), p. 120-127.

[7] This work was supported by the National Research Foundation of Korea (NRF) grant funded by the Korea government (MSIP) (No. 2015R1A2A2A01006992), Nano Material Technology Development Program (2012M3A7B4049807) and IBS-R019-D1.
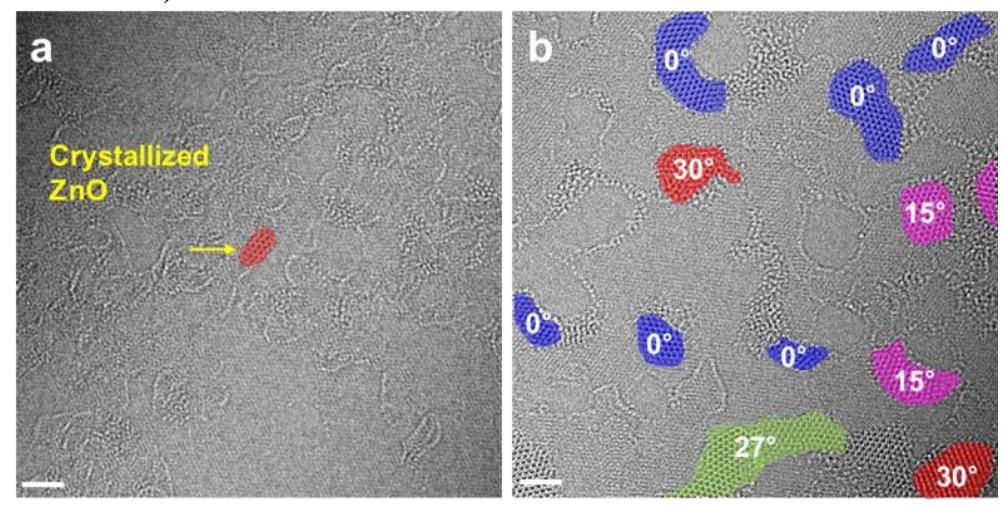

Figure 1. $\mathrm{ZnO}$ monolayer on pristine and UV/ozone-treated graphene. (a) Atomic resolution image of $\mathrm{ZnO}$ nanocluster on graphene. (b) Atomic resolution image of $\mathrm{ZnO}$ nanoclusters on a graphene substrate with $180 \mathrm{~s}$ of UV/ozone treatment.

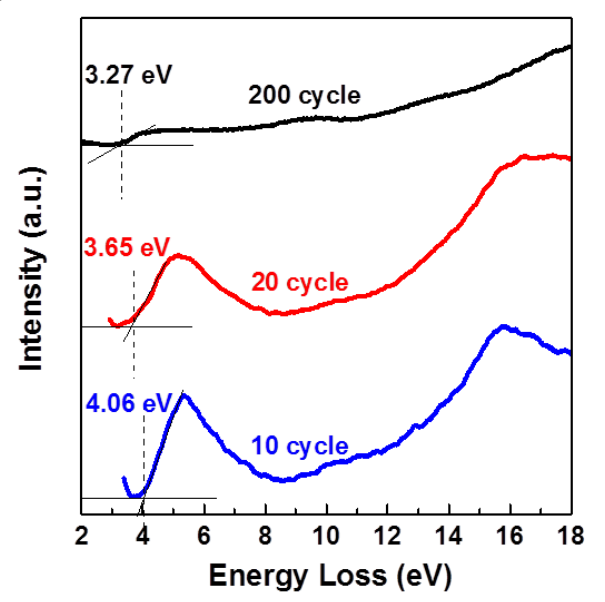

Figure 2. STEM-EELS spectra of $\mathrm{ZnO}$ deposited with different ALD cycles on UV/ozone-treated graphene. 